\title{
Diagnostic génétique préimplantatoire : législation et aspects éthiques
}

\author{
Stéphane Viville, Pierre Ray, Christiane Wittemer, \\ Jeanine Ohl, Pierre Dellenbach, Pierre Gerlinger
}

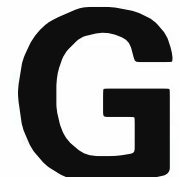
râce aux progrès récents de la biologie moléculaire et des techniques d'assistance à la procréation, il est maintenant possible de proposer à un couple, présentant un haut risque de transmettre une maladie génétique d'une particulière gravité, de caractériser la mutation responsable avant l'implantation des embryons et ainsi de leur éviter une interruption médicale de grossesse (IMG) et ses conséquences psychologiques [1]. En effet, le diagnostic génétique préimplantatoire (DPI) permet de caractériser le statut génétique des embryons obtenus par fécondation in vitro (FIV) et donc de ne transférer que des embryons sains ou porteurs hétérozygotes [2-4]. Ce tri des embryons avant réimplantation est clairement plus facile à accepter qu'une IMG, aussi précoce soit-elle.

Cette technique est à considérer comme une forme de diagnostic prénatal précoce. Une telle approche de la médecine préventive n'est pas sans soulever des problèmes d'ordre éthique qui sont liés au tri des embryons. Ces problèmes concernent non seulement le généticien, le biologiste, le médecin mais aussi l'homme politique car il convient de proposer une approche cohérente et acceptable pour tous. Dans cet article, nous aborderons l'aspect

\section{La législation française}

Après un long débat, la France s'est dotée d'une législation autorisant le diagnostic génétique préimplantatoire ou tout du moins ne l'interdisant pas. Cette pratique devrait pouvoir être réalisée mais sous un strict contrôle. La loi $\mathrm{Ln}^{\circ}$ 94-654 du 29 juillet 1994 relative au don et à l'utilisation des éléments et produits du corps humain, à l'assistance médicale à la procréation et au diagnostic prénatal, ainsi que les décrets d'application $n^{\circ} 95-558$ à 560 du 6 mai 1995 définissent les conditions dans lesquelles peuvent se pratiquer le DPI. Cependant, certains textes sont toujours en attente de leur décrets d'application.

L'article L. 162-17 stipule que "Le diagnostic biologique effectué à partir de cellules prélevées sur l'embryon in vitro n'est autorisé qu'à titre exceptionnel dans les conditions suivantes:

Un médecin exerçant son activité dans un centre de diagnostic prénatal pluridisciplinaire tel que défini par l'article L. 162-16 doit attester que le couple, du fait de sa situation familiale, a une forte probabilité de donner naissance à un enfant atteint d'une maladie génétique d'une particulière gravité, reconnue comme incurable au moment du diagnostic. Le diagnostic ne peut être effectué que lorsque a été préalablement et précisément identifié, chez l'un des parents, l'anomalie ou les anomalies responsables d'une telle maladie.

Les deux membres du couple expriment par écrit leur consentement à la réalisation du diagnostic.

Le diagnostic ne peut avoir d'autre objet que de rechercher cette affection ainsi que les moyens de la prévenir et de la traiter.

Il ne peut être réalisé que dans un établissement spécifiquement autorisé à cet effet après avis de la Commission Nationale de Médecine et de Biologie de la Reproduction et du Diagnostic Prénatal et dans les conditions définies par décret en Conseil d'État ".

L'article L.162-16 auquel il est fait référence dans l'article L.162-17 précise les conditions de la pratique du diagnostic prénatal: "seuls des établissements publiques de santé et des laboratoires d'analyses de biologie médicales après autorisation délivrée par arrêté du ministre chargé de la santé peuvent pratiquer de tels diagnostics ".

L'article L.152-2 précise que l'assistance médicale à la procréation est destinée à répondre à la demande parentale d'un couple. "Elle a pour objet de remédier à l'infertilité dont le caractère pathologique a été médicalement diagnostiqué. Elle peut avoir pour objet d'éviter la transmission à l'enfant d'une maladie d'une particulière gravité. " 
L'article L.152-8, qui est relatif à l'assistance médicale à la procréation pose actuellement un problème quant à la réalisation du DPI. En effet, il précise que «la conception d'embryons humains à des fins d'étude, de recherche ou d'expérimentation est interdite.

Toute expérimentation sur l'embryon est interdite.

A titre exceptionnel, l'homme et la femme formant le couple peuvent accepter que soit menées des études sur leurs embryons.

Leur décision est exprimée par écrit. Ces études doivent avoir une finalité médicale et ne peuvent porter atteinte à l'embryon".

Les décrets n ${ }^{\circ}$ 5-558 à 560 précisent les conditions dans lesquelles le diagnostic prénatal (DPN) et le DPI peuvent être pratiqués mais aucun décret précisant l'application des articles L.152-2 et L.152-8 n'a encore été publié.

Ces décrets concernent aussi la composition, l'organisation, l'attribution et le fonctionnement de la Commission Nationale de Médecine et de Biologie de la Reproduction et du Diagnostic Prénatal. Celle-ci est composée d'une quarantaine de membres, dix membres de droit du fait de leur responsabilité au sein de l'organisation de la santé en France et une trentaine de membres nommés pour trois ans par le ministère chargé de la Santé en fonction de leur compétence dans les domaines impliqués par le DPI.

Elle a pour attribution de donner au ministre chargé de la santé des avis motivés sur les demandes d'agréments pour les actes cliniques et biologiques d'assistance médicale à la procréation, pour la pratique du diagnostic prénatal ainsi que pour pratiquer «les activités de diagnostic biologique à partir de cellules prélevées sur l'embryon in vitro ". C'est elle aussi qui donne son avis sur les renouvellements ou les retraits de ces agréments. De plus, elle remet chaque année au ministère chargé de la Santé un rapport portant sur l'évolution de la médecine et de la biologie de la reproduction et du diagnostic prénatal. Les centres autorisés sont tenus de présenter au ministère chargé de la Santé un rapport annuel d'activité en vue de leur évaluation à laquelle la commission participe.

D'après les textes mentionnés il est clair que le législateur a souhaité lier le DPI à la pratique du diagnostic prénatal. Cela semble logique dans la mesure où le DPI est à considérer comme une forme précoce du diagnostic prénatal. En revanche, l'utilisation des termes «à titre exceptionnel » et «incurable au moment du diagnostic" n'apparaissent pas pour le diagnostic prénatal de même que la nécessité d'avoir préalablement et précisément identifié l'anomalie responsable de la maladie. Cela impose des restrictions comme l'impossibilité de tester un embryon sans avoir identifié la ou les mutations chez les parents qui ne souhaitent pas savoir s'ils sont atteints [5]. Cette attitude reflète probablement la crainte qu'inspire cette pratique en France.

Il est à noter que la loi ne stipule pas que cette pratique doit se faire dans une unité d'assistance médicale à la procréation mais, pour des raisons pratiques, puisque le DPI est dépendant de la fécondation in vitro (FIV) et pour des problèmes techniques, ce dernier se fera dans de telles unités. La loi ne parle pas de diagnostic génétique préimplantatoire mais de diagnostic biologique à partir de cellules prélevées sur l'embryon in vitro, cela afin de considérer toutes les formes de diagnostic possible. Dans les faits, seules les techniques de génétique permettent d'obtenir une sensibilité suffisante pour établir un diagnostic à partir d'une ou de deux cellules prélevées sur un embryon in vitro. Mais il n'est pas à exclure que des méthodes de diagnostic biochimiques atteignent un jour une telle sensibilité $[6,7]$.

Il reste une ambiguité dans la loi concernant le DPI. En effet, il est précisé (article L.152-8) que les études sont interdites sur l'embryon humain sauf à titre exceptionnel, avec l'autorisation du couple, seulement si ces études ont un but médical et si elles ne portent pas atteinte à l'embryon. Cette dernière phrase est sujette à différentes interprétations. Or, même s'il y a atteinte à l'intégrité de l'embryon, les travaux réalisés aussi bien chez l'animal que chez l'homme montrent que le prélève- ment d'une ou de deux cellules au stade huit cellules n'affecte pas, en général, le développement de l'embryon [8-12] En revanche, comme la congélation d'embryons, qui est bien acceptée, conduit à la perte d'un certain nombre d'embryons, il faut être prêt à l'accepter également pour le DPI. Cette notion limite aussi les possibilités de mise au point des techniques nécessaires sur l'embryon humain. Il faudra admettre comme suffisants les travaux réalisés sur l'animal, la souris par exemple [13].

Il est actuellement nécessaire d'attendre que soit précisée la signification de ce textc par la publication de son décret d'application.

La France s'est dotée d'une législation forte, ce qui n'est pas le cas d'autres pays européens. Ainsi, pour l'Angleterre, où fut développée cette technique $[14,15]$, et pour l'Espagne, la législation est très permissive et non spécifique au DPI: toute recherche sur l'embryon humain est autorisée, puisqu'il existe en Angleterre et en Espagne une notion de pré-embryon, jusqu'au $14^{\mathrm{c}}$ jour du développement. Le DPI est soumis à une autorisation préalable délivrée par un comité d'éthique. Le DPI n'est donc pas directement mentionné dans la législation anglaise mais reste sous le contrôle d'un comité d'éthique qui statue sur l'ensemble des recherches menées sur l'homme. La Belgique n'a toujours pas de législation à ce propos alors que l'Allemagne interdit tout forme de recherche sur l'embiryon humain et donc le DPI.

\section{Le diagnostic préimplantatoire pour qui?}

Comme le stipule la loi, le DPI s'adresse à des couples qui, du fait de leur situation familiale, ont une forte probabilité de donner naissance à un enfant atteint d'une maladie génétique d'une particulière gravité reconnue comme incurable au moment du diagnostic. De plus, ce diagnostic ne peut être effectué que lorsqu'a été préalablement et précisément identifiée, chez au moins l'un des parents concernés, l'anomalie responsable. Mais aucune liste de 
maladies concernées, considérée comme acceptable, n'a été établie. Cela nous permet de soulever les différents problèmes que pose cette notion de maladie gravissime incurable au moment du diagnostic. En effet, si cette notion est claire pour des maladies comme la myopathie de Duchenne ou la mucoviscidose où l'espérance de vie de l'enfant est réduite, elle devient moins facile à apprécier pour des maladies dont l'apparition est tardive, par exemple, pour la chorée de Huntington ou la maladie d'Alzheimer. La question est de savoir si dans de tels cas le droit au DPI est légitime. De plus, la nécessité de caractériser la mutation chez les parents peut être mal vécue. Or il est possible, grâce aux séquences microsatellites polymorphes et dans la mesure où les grands parents de l'enfant à naître sont toujours en vie, d'identifier l'origine des allèles chez les embryons. Cela permet de ne transférer que les embryons porteurs des allèles des grands parents sains, sans pour autant déterminer le statut génétique des parents. Mais dans ce cas, comme lors de la détermination du sexe des embryons pour les maladies liées aux chromosomes $\mathrm{X}$, un quart des embryons rejetés sont sains. Cela nécessite aussi d'avoir accès aux grands-parents. Schulman et al. (Fairfax, VA, USA) suggèrent, quant à eux, d'effectuer les diagnostics sur les embryons sans divulguer la moindre information aux couples traités [5]. Le problème est encore plus délicat pour les gènes de susceptibilité à une maladie puisqu'il est clairement établi que l'environnement a une influence non négligeable sur le développement de la maladie.

Nous pensons que dans un premier temps seules les maladies d'une particulière gravité, incurables et touchant le jeune enfant doivent être envisagées. Pour les maladies telles que la chorée de Huntington ou la maladie d'Alzheimer le débat reste ouvert (au moins en France). En effet, il n'est pas exclu que d'ici 40 à 60 ans, ce qui correspond à l'âge moyen d'apparition de ces syndromes, un traitement soit disponible. De plus, nous pourrions multiplier les exemples de vies passionnantes interrompues bien oublier les angoisses des parents et nous rappelons que certaines équipes offrent la possibilité d'avoir recours à une IMG dans les cas de la chorée de Huntington.

La pratique du DPI implique aussi la possibilité d'offrir accès aux techniques de l'assistance médicale à la procréation à des couples fertiles. Or celles-ci, du fait des échecs successifs fréquents, ne sont pas sans conséquences psychologiques. Mais il est probable que les couples demandeurs d'un DPI, surtout lors de maladies gravissimes, auront une motivation telle qu'ils seront aptes à surmonter les difficultés liées à la FIV.

\section{Problèmes eugéniques posés par le DPI}

Des voix se sont fait entendre à propos des dérives eugéniques éventuelles de la pratique du DPI. Cette pratique a même été présentée comme l'ultime forme médicale de l'eugénisme [16], point de vue qui a fait vivement réagir la communauté scientifique internationale [17-19].

De pratique ancienne l'eugénisme fut élevé au rang de "science" à la fin du siècle dernier. Ce fut Francis Galton, cousin de Darwin, qui vers 1870 créa "l'eugénisme scientifique » qui a selon lui deux buts: l'un est d'empêcher la multiplication des inaptes, c'est l'eugénisme négatif; l'autre est de favoriser la multiplication des plus aptes afin d'améliorer la race humaine, c'est l'eugénisme positif. Cette "science» conduira un grand nombre de gouvernements à adopter des programmes de stérilisation des inaptes. C'est aussi l'eugénisme qui servit de justification aux horreurs pratiquées par les nazis au cours de la dernière guerre.

Le Dr J. Testard dans son livre «Le désir du gène " (Flammarion, Paris, 1994) dans lequel il s'oppose farouchement au DPI, explique qu'un "eugénisme médicalisé " se met actuellement en place. Selon lui la sélection des "bons gènes» (eugénisme positif) et des "mauvais gènes" (eugénisme négatif) se fait au niveau de l'embryon. L'argumentation développée dans ce livre ainsi que dans un article plus récent [16] est fondée sur deux points principaux. Le premier est d'ordre technique. Il est avancé que les progrès de la biologie moléculaire, les techniques de FIV et la possibilité de prélever un ou deux blastomères sur un embryon humain sans en affecter son devenir permettent actuellement d'identifier un gène et bientôt une dizaine d'entre eux sur des embryons humains et donc de les trier. Le deuxième argument qui, selon le Dr Testard, va nous conduire à "un eugénisme médicalisé " est le désir des parents d'avoir un enfant parfait. Un tel désir devrait pousser les futurs parents à demander la caractérisation du contenu génomique de leur futur enfant à l'aide des techniques utilisées par le DPI. Il va de soi qu'à ce stade il ne s'agit plus d'une technique devant mener à une pratique diagnostique ou thérapeutique mais d'un service et que ce raisonnement poussé à cette extrémité ne concerne plus un problème médical.

Nous avons plusieurs arguments à opposer. D'abord au niveau technique, il est vrai que dans un temps très proche il sera possible d'examiner un nombre important de gènes à partir d'une seule cellule, de l'ordre de 10 à 20. Mais cela est faible en regard des 75000 à 100000 gènes différents que contient notre génome. De plus, cet ensemble de gènes forme une combinatoire d'une complexité telle que peu de programmes informatiques seraient aptes à la gérer. Il est donc peu imaginable que dans un avenir proche nous ayons les connaissances suffisantes pour espérer sélectionner, puisque c'est de cela qu'il s'agit, le surhomme des fantasmes souvent exprimés dans la littérature et le cinéma. Enfin, en imaginant qu'une équipe accepte de le faire, il est peu probable que les futurs parents acceptent de passer par les désagréments d'un protocole de FIV pour pouvoir choisir le sexe ou la couleur des yeux de leur enfant. Il est bon de rappeler que, si la FIV représente sans conteste possible un progrès quant au traitement des stérilités féminines et masculines, elle n'en reste pas moins un procédé long et douloureux. Seuls des couples motivés acceptent un tel protocole. Outre les problèmes techniques, il faut considérer le but final de l'eugé- 
nisme qui, nous le rappelons, consiste à l'amélioration de l'espèce humaine. Pour ce faire, il faudrait dans un premier temps envisager l'éradication de l'ensemble des mutations à l'échelle de la population terrestre. Cela est matériellement impossible, en tout premier lieu à cause de l'apparition d'un nombre élevé de néomutations et ensuite par le gigantisme du contrôle des naissances que cela impliquerait! L'aspect statistique de l'eugénisme ne doit pas être oublié; espérer influencer l'évolution de l'espèce humaine impliquerait de contrôler la majorité des naissances.

Notons, sans entrer dans l'éternel débat concernant l'inné et l'acquis, que le DPI est proposé, actuellement, uniquement pour des maladies monogéniques graves, sans espoir pour l'enfant à naître et cela quel que soit son environnement.

Il nous semble assez dérisoire de s'opposer au DPI, y compris par des méthodes de détermination du sexe des embryons qui impliquent le rejet d'embryons sains, alors que l'avortement pour convenance personnelle est bien accepté dans notre société

\section{RÉFÉRENCES}

1. Pergament E. Preimplantation diagnosis: a patient perspective. Prenat Diagn 1991 ; 11: 493-500.

2. Handyside A. Diagnosis of inherited disease before implantation. Reprod Med Rev $1993 ; 2: 51-61$.

3. Black SH. Preimplantation genetic diagnosis. Curr Op Pediatr 1994; 6: 712-6. 4. Delhanty JD. Preimplantation diagnosis.
Prenat Diagn 1994; 14:1217-27.

5. Schulman JD, Black SH, Handyside A, Nance WE. Preimplantation genetic testing for Huntington disease and certain other dominantly inherited disorders. Clin Genet $1996 ; 49: 57-8$.

$\mathrm{m} / \mathrm{s} n^{\circ} 12$, vol. 12, décembre 96
6. Sermon K, Lissens W, Tarlatzis B, Braude PR, Devroey P, Van Steirteghem A, Liebaers I. Beta-N-acetylhexosaminidase activity in human oocytes and preimplantation embryos. Hum Reprod 1992; 7: 1278-80.

7. Epstein M, Avital Y, Agmon V, Dinur T, Fibach E, Gatt S, Laufer N. Diagnosing sphingolipidoses in murine and human embryos. Hum Reprod 1993; 8: 302-9.

8. Hardy K, Martin KL, Leese HJ, Winston RM, Handyside AH. Human preimplantation development in vitro is not adversely affected by biopsy at the 8-cell stage. Hum Reprod $1990 ; 5: 708-14$.

9. Tarin JJ, Conaghan J, Winston RM, Handyside AH. Human embryo biopsy on the 2nd day after insemination for preimplantation diagnosis : removal of a quarter of embryo retards cleavage. Fertil Steril 1992; 58: 970-6.

10. Cui KH, Pannall P, Cates G, Matthews $\mathrm{CD}$. Blood analysis of mice born following single-cell embryo biopsy. Hum Reprod $1993 ; 8: 1906-9$.

11. Liu J, Van den Abbeel E, Van Steirteghem $\mathrm{A}$. The in-vitro and in-vivo developmental potential of frozen and non-frozen biopsied 8-cell mouse embryos. Hum Reprod 1993 ; 8 : 1481-6.

12. Cui KH, Barua R, Matthews CD. Histopathological analysis of mice born following single cell embryo biopsy. Hum Reprod 1994; 9: 1146-52.

13. Ji YZ, Nicolas JC, Marechal V, Desire N, Pavirani A, Milliez J. Diagnostic préimplantatoire de la mutation deltaF508 de la mucoviscidose chez la souris transgénique. Presse Med 1995; 24: 1193-7.

14. Handyside AH, Pattinson JK, Penketh RJ, Delhanty JD, Winston RM, Tuddenham EG. Biopsy of human preimplantation embryos and sexing by DNA amplification. Lancet $1989 ; 1$ : 347-9.

15. Handyside $\mathrm{AH}$, Kontogianni $\mathrm{EH}$, Hardy K, Winston RM. Pregnancies from biopsied human preimplantation embryos sexed by Y-specific DNA amplification. Nature 1990; 344: 768-70.

16. Testard J, Sèle B. Towards an efficient medical eugenics: is the desirable always the feasible. Hum Reprod 1995; 10 : 3086-90.

17. Schulman JD, Edwards RG. Preimplantation diagnosis is disease control, not eugenics. Hum Reprod 1996; 11 : 463-4.

18. Handyside A, Commonsense as applied to eugenics: response to Testart and Sèle. Hum Reprod 1996; 11 : 707.

19. Fiddler M, Pergament E. Medically-assisted procreation: a maturing technology or a premature fear? Response to Testart and Sèle. Hum Reprod 1996; 11 : 708-9.

\section{S. Viville}

Assistant hospitalier universitaire. Service de biologie de la reproduction hopitaux universitaires de Strasbourg et IGBMC 1, rue Laurent-Fries, BP163, 67404 Illkirch CU de Strasbourg Cedex, France.

\section{P. Ray}

Étudiant en thèse, Human Embryology Laboratory, Institute of Obstetrics and Gynaecology, Royal Postgraduate Medical School, Hammersmith Hospital, Du Cane Road, Londres W12 ONN, Grande-Bretagne.

\section{Wittemer} Biologiste.

\section{J. Ohl}

Docteur en médecine, assistant.

\section{P. Dellenbach}

Professeur des universités, praticien hospitalier, département de procréation médicalement assistée, Service de gynécologie obstétrique, CMCO, 19, rue Louis-Pasteur, BP120, 67303 Schiltigheim Cedex, France.

\section{P. Gerlinger}

Professeur des universités, praticien hospitalier, Service de biologie de la reproduction hôpitaux universitaires de Strasbourg, 1, place de l'Hôpital, 67000 Strasbourg, France.

\section{Remerciements}

S. Viville tient à remercier le Dr A Handyside pour les fructueuses discussions qu'ils ont pu avoir sur le sujet. Nous remercions les Drs Georges Imbert et Brigitte Viville pour leur lecture critique de ce manuscrit.

\section{TIRÉS À PART}

S. Viville. 